\title{
Defining System Boundaries of an Institution Nitrogen Footprint
}

\author{
Elizabeth de la Reguera, ${ }^{1}$ Elizabeth A. Castner, ${ }^{2}$ James N. Galloway, ${ }^{2}$ Allison M. Leach, ${ }^{3}$ \\ Neil Leary, ${ }^{4}$ and Jianwu Tang ${ }^{1}$
}

\section{Abstract}

A nitrogen (N) footprint quantifies the amount of reactive nitrogen released to the environment and can be measured at different scales. The $N$ footprint of a university includes activities and consumption within its geographic boundaries as well as activities that support the institution. Determining system bounds of an $N$ footprint depends on the institution's mission and provides a common baseline for comparison. A comparison of three scopes of the $N$ footprint, which describe how emissions are directly related to an institution's activities, was conducted for seven institutions. Scopes follow the established definition for the carbon footprint. In this article, the authors propose a new system bounds definition (core campus versus adjunct). Two case studies were explored: how the N footprint of Dickinson College changed with air travel, and how the N footprint of the Marine Biological Laboratory changed with scientific research. Of the three scopes, scope 3 was consistently the largest proportion of the $N$ footprint for all seven institutions. The core campus activities of Dickinson College made up 99 percent of its $N$ footprint, with air travel making up the remaining 1 percent. The Marine Biological Laboratory's core campus activities made up 51 percent of its $N$ footprint and the scientific research made up the remaining 49 percent. Institutions should define system bounds based on their mission and stay consistent with their boundaries following the baseline year. The core campus footprint could be used to compare institution footprints using consistent system bounds. How institutions define their boundaries will impact the recorded amount of nitrogen as well as how the institution will set reduction strategies.

Keywords: adjunct footprint; core campus footprint; nitrogen footprint; scopes; system boundaries

\section{Introduction}

A nitrogen $(\mathrm{N})$ footprint quantifies the amount of reactive nitrogen released to the environment as a result of an entity's resource consumption. ${ }^{1}$ Reactive nitrogen $(\mathrm{Nr})$ is defined as all nitrogen except nitrogen gas $\left(\mathrm{N}_{2}\right){ }^{2}$ Humans create reactive nitrogen by breaking the triple bond of $\mathrm{N}_{2}$ through the Haber-Bosch process, the cultivation of legumes, and the combustion of fossil fuels. ${ }^{3}$ The detrimental impacts to ecosystems and human health caused by $\mathrm{Nr}$ indicate the importance of managing nitrogen emissions in order to reduce its impact on the environment.

This article examines how changing system boundaries affects the $\mathrm{N}$ footprint results of different institutions. Setting system boundaries is important because it provides a common baseline for comparison as well as allows different entities within an institution to have direct interaction with their footprint. The following topics are addressed:

1. The scopes for the $\mathrm{N}$ footprints of seven institutions are compared.

2. A new system bounds definition is proposed for comparing institutions: the core campus footprint

'Marine Biological Laboratory, The Ecosystems Center, Woods Hole, Massacheusetts.

${ }^{2}$ Department of Environmental Sciences, University of Virginia, Charlottesville, Virginia.

${ }^{3}$ Department of Natural Resources \& the Environment, The Sustainability Institute, University of New Hampshire, Durham, New Hampshire.

${ }^{4}$ Center for Sustainability Education, Dickinson College, Carlisle, Pennsylvania.

(c) Elizabeth de la Reguera et al., 2017; Published by Mary Ann Liebert, Inc. This Open Access article is distributed under the terms of the Creative Commons Attribution Noncommerical License (http://creativecommons.org/licenses/by-nc/4.0), which permits any noncommercial use, distribution, and reproduction in any medium, provided the original author(s) and the source are credited. 
versus the adjunct footprint, illustrated by results from six institutions.

3. Two case studies for specific institutions are explored. Case Study A analyzes how the $\mathrm{N}$ footprint of Dickinson College changes with the inclusion of air travel. Case Study B assesses how the N footprint of the Marine Biological Laboratory changes with scientific research.

The various system bounds discussed in this article focus on the scopes, the core campus activities, other components of energy consumption, and research activities.

\section{Methods}

\section{System Bounds and Scopes}

The $\mathrm{N}$ footprint could be measured at different scales, from an individual person to institutions, regions, and the globe. ${ }^{1,3,4,5}$ The $\mathrm{N}$ footprint of an institution, such as a university, includes activities and consumption within the geographic boundaries of the institution as well as supporting activities and consumption that occur outside the boundaries (e.g., food production, commuting, electricity generation). ${ }^{3}$ To calculate an institution's $\mathrm{N}$ footprint, the system bounds must be clearly and carefully designed. Setting boundaries determines what will be included in the footprint. For example, in tracking carbon dioxide $\left(\mathrm{CO}_{2}\right)$ emissions, the Climate Registry describes the parameters used for defining the boundaries, which include scopes, gases, activity types (e.g., stationary combustion), and geographic/operation boundaries. ${ }^{6}$

One framework for determining systems bounds is to allocate emissions into three scopes based on where they occur relative to the given institution as well as how directly those emissions are related to the institution's activities. Scopes are groupings of sectors that account for emissions that occur as a result of direct or indirect activity by the institution. The sectors allocated to scopes are defined by the World Resources Institute and the World Business Council on Sustainable Development. ${ }^{7}$ Both the carbon footprint tool for institutions (the Carbon Management and Assessment Platform) and the $\mathrm{N}$ footprint tool collect data for scopes 1 through $3{ }^{8}$ Scope 1 includes direct emissions that occur from institution-ownedand-operated equipment. In scope 1 , both footprints report on-campus stationary sources, direct transportation sources, research animals, and the amount of fertilizer applied to landscape. Scope 2 accounts for indirect emissions from purchased utilities; both footprints report purchased electricity. Finally, scope 3 includes losses from other indirect consequences of institutional activities and consumption patterns (e.g., commuting and food production) that may not necessarily occur within institution-owned equipment (e.g., wastewater).

For the carbon footprint tool, scope 3 is considered optional. Institutions that have signed the American College and University Presidents' Climate Commitment (ACUPCC) are required to report scope 3 emissions for commuting and air travel that is paid for by the institution. There are many types of scope 3 emissions. The consumption associated with these emissions is often not collected in a centralized way, making them difficult to track and report. Some institutions determine which parts of scope 3 to include based on finances or control boundaries. ${ }^{9}$
In contrast, all institutions in the $\mathrm{N}$ footprint network include scope 3 in their calculations and have included food purchases in their footprint. Including food in the $\mathrm{N}$ footprint is important because virtual nitrogen (any nitrogen used in the food production process but not contained in the food we consume) associated with food is one of the largest contributors of reactive nitrogen for most higher education institutions. ${ }^{1}$ In addition, many activities in scope 3 (such as food purchases) are necessary to support an institution's operations. Given the significance of scope 3 , it is important to identify other methods of defining system bounds that better emphasize relevant environmental impacts that are also integral to an institution's activities.

The $\mathrm{N}$ footprints for the seven institutions that completed the calculation were compared by scope. Each institution similarly calculated its $\mathrm{N}$ footprint by following methods established by the Nitrogen Footprint Tool. ${ }^{10}$ The average proportion for each scope was calculated, and the contribution and importance of scope 3 was considered for the $\mathrm{N}$ footprint.

\section{Core Campus Footprint versus Adjunct Footprint}

Another way of defining system boundaries is by distinguishing core facilities and activities from adjunct facilities and activities. For example, a university may have a main campus at which its core undergraduate and graduate education activities are conducted, a separate facility that is primarily research and not an education facility, a hospital, and/or a farm. The core footprint encompasses the main campus and its educational activities, while an adjunct footprint 
encompasses the other facilities and activities.

The core campus footprint includes any activity that occurs on the campus and contributes to the core functioning of that institution. It also includes the upstream emissions associated with the production or distribution of that activity. The core campus footprint supports baseline institutional activities, including oncampus energy usage, public transit, commuting, fertilizer, and food. The adjunct footprint includes all off-campus research activities that are not directly related to the core campus activities. It should be noted that some research is encompassed within the core campus footprint; the adjunct footprint focuses on major off-campus research initiatives. Through using scopes to determine the system bounds of both core campus footprints and adjunct footprints, institutions can capture the full depths of each scope within the campus. The distinction between the two footprints will assist institutions in setting reduction strategies appropriate for each footprint.

This article compares the two levels of footprint system bounds (core campus footprint and adjunct footprint) of six institutions, with a focus on case studies for Dickinson College and the Marine Biological Laboratory. In addition, it explores a specific example of research activities for each institution.

\section{Case Study A: Dickinson College and Air Travel}

Dickinson College, a small residential liberal arts school located in Carlisle, Pennsylvania, emphasizes global study, sustainability, and civic engagement. The first $\mathrm{N}$ footprint was calculated for the period August 2011 through July 2012 as a senior undergraduate thesis, and was updated for August 2013 through July $2014 .^{11,12}$ In addition, Dickinson reports its carbon footprint annually to the ACUPCC and has set reduction goals to reduce greenhouse gas (GHG) emissions 25 percent from the 2008 level by 2020, and to reach net zero emissions in that same year.

Dickinson set its boundaries based on the boundaries used in its carbon footprint. Dickinson owns a college farm where some food grown is supplied to the college and the remainder is sold to Community Supported Agriculture members or at the local farmers' market. The farm operations that were included in the $\mathrm{N}$ footprint were all college food purchases from the farm, fertilizer purchased by the farm, and all fuels and electricity used.

For Dickinson, accounting for air travel included any air travel directly financed by the school. The Campus Carbon Calculator ${ }^{\mathrm{Tix}}$ allows for the accounting of "directly encouraged emissions," which include study abroad air travel and regular commuting by faculty, staff, and students. If Dickinson was directly financing a visiting faculty member or student, then their travels were included in the footprint.

Air travel is a scope 3 activity that not all institutions track. The Dickinson footprint was compared with and without air travel.

\section{Case Study B: Marine Biological Laboratory and Scientific Research}

The Marine Biological Laboratory $(\mathrm{MBL})$ is a private nonprofit scientific research institution affiliated with the University of Chicago and located in Woods Hole, Massachusetts. It has a small number of yearround employees (approximately 250 ), but in the summer season the MBL grows in size due to visiting scientists and students. The first $\mathrm{N}$ footprint was calculated for the period January to December 2011 as a research project for the Semester in Environmental Science; the footprint was updated for January to December 2013. ${ }^{13,14}$

The MBL set its system boundaries based on the geographic bounds of its campus. Although MBL has numerous field sites, they were excluded because experiments are temporary and only a few MBL employees reside at field sites. In addition, these scientific activities were not included in the core campus footprint (e.g., fertilization experiments) because setting a reduction goal does not include altering the research of scientists.

The scenario unique to the MBL is its research activities. Therefore, its footprint was compared with and without research activities in their base year.

\section{Results and Discussion}

\section{Scopes}

Of the three scopes, scope 3 is consistently the largest proportion of each institution's $\mathrm{N}$ footprint (Figure 1). On average, scope 3 contributes 70 percent of the $\mathrm{N}$ footprint for the seven institutions that have calculated their footprint. The range is from 37 percent to 87 percent. Scope 2 is the second-largest contributor, making up an average of 22 percent (range: $4 \%-50 \%$ ). Scope 1 has the smallest contribution, averaging just 7 percent (range: $3 \%$ $12 \%$ ) of the total $\mathrm{N}$ footprint. 


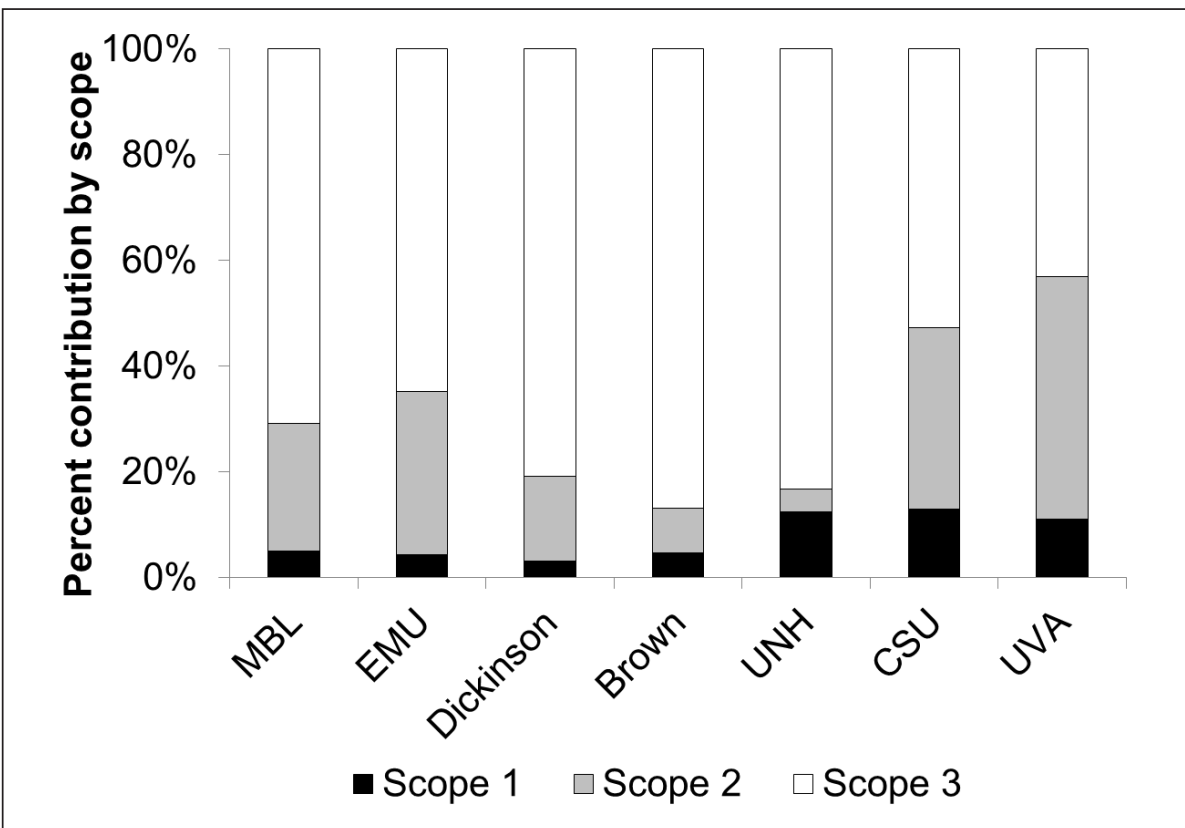

Figure 1. The percent contribution of scopes 1, 2, and 3 to the $\mathrm{N}$ footprint of seven institutions: the Marine Biological Laboratory (MBL), Eastern Mennonite University (EMU), Dickinson College, Brown University, University of New Hampshire (UNH), Colorado State University (CSU), and the University of Virginia (UVA) ${ }^{14}$

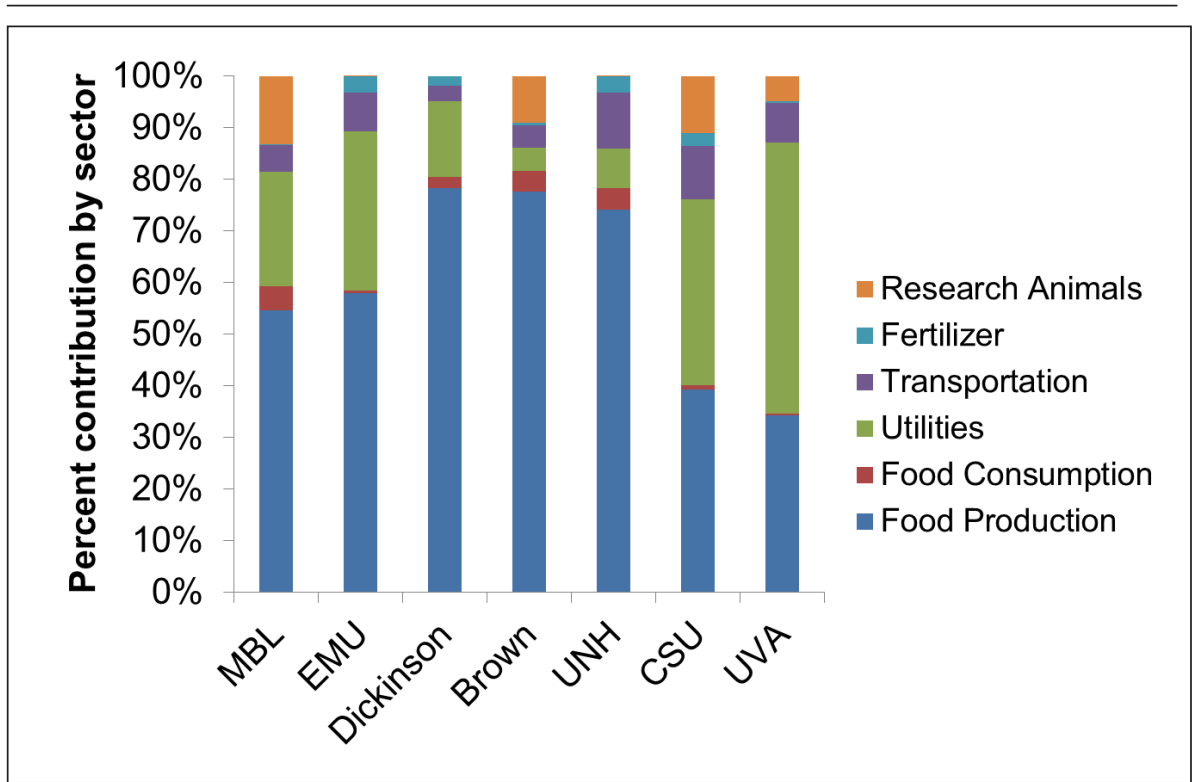

Figure 2. The percent contribution of six sectors to the nitrogen footprint of seven institutions: the Marine Biological Laboratory (MBL), Eastern Mennonite University (EMU), Dickinson College, Brown University, University of New Hampshire (UNH), Colorado State University (CSU), and the University of Virginia (UVA) ${ }^{14}$

According to carbon footprint accounting protocols, only scopes 1 and 2 must be calculated and reported-scope 3 is optional. If only scopes 1 and 2 were reported for the $\mathrm{N}$ footprint, then just 30 percent of the total $\mathrm{N}$ footprint would be accounted for. Food production makes up the largest proportion of scope 3. By including this scope, an important sector that makes up the majority of all calculated $\mathrm{N}$ footprints can be included (Figure 2).

\section{Core Campus Footprint} versus Adjunct Footprint

In addition to their main campus, institutions often have research facilities such as a hospital, veterinary school, or agricultural facilities. These institutionally owned operations can be large enough that they have their own geographical boundaries and activities that span all three scopes and most of the sectors. However, their activities are focused on research, which is often part of the mission of an institution.

The contributions of the core footprint versus the adjunct footprint can vary widely based on an institution's activities (Figure 3). Two examples that represent the extremes of institution focus are Dickinson College and the MBL (Figure 4). Dickinson is an undergraduate college with limited research, whereas the MBL is a research institution with limited coursework. The footprints reflect these activities. The core campus activities make up 99 percent of the Dickinson $\mathrm{N}$ footprint but just 51 percent of the MBL $\mathrm{N}$ footprint. Adjunct facilities make up almost half (49\%) of the $\mathrm{N}$ footprint for MBL when all research activities are accounted for.

Calculating a footprint focusing just on the core campus activities initially is beneficial because it can make comparing footprints across institutions more comparable. Comparing institutions with different system bounds can lead to misleading or erroneous conclusions. The core campus activities footprint resolves this issue by focusing on activities consistent across institutions. In addition, reduction strategies that are focused on core campus activities will not affect an institution's mission related to research. 


\section{Case Study A: Dickinson College and Air Travel}

Dickinson College strongly encourages students to study abroad and take advantage of the global education opportunities it offers. Roughly 60 percent of Dickinson students will study abroad during their academic career and more than 40 percent of faculty members have led a study abroad program. ${ }^{15}$ Other air travel associated with Dickinson's campus is athletic teams traveling for games and tournaments and faculty members traveling for research and development. Although air travel is a small portion of Dickinson's overall footprint, it is part of the overall educational mission and is included in its carbon footprint. Therefore air travel was included in the $\mathrm{N}$ footprint. Dickinson in 2014 released a total of 85 metric tons $\mathrm{N}$, which included all air travel (for study abroad, athletics, and research and development). (See Figure 5.) Air travel of all three types totaled approximately 1.3 metric tons $\mathrm{N}$, which is about 1.5 percent of total nitrogen released by Dickinson. By removing air travel from the footprint, the total dropped by 1.3 metric tons $\mathrm{N}$, to 84 .

Many institutions base their $\mathrm{N}$ footprint calculations on annual per person totals. In order to ensure a uniform comparison, Dickinson calculated its total kilograms of nitrogen released per person annually and found that for food production, food consumption, utilities, and fertilizer, nitrogen per person was 22, $0.57,4.1,0.52$ metric tons $\mathrm{N}$, respectively. When excluding air travel, transportation releases were $0.40 \mathrm{~kg}$ $\mathrm{N} /$ person; when including air travel the release of nitrogen per person doubled to $0.82 \mathrm{~kg} \mathrm{~N} /$ person. Al-

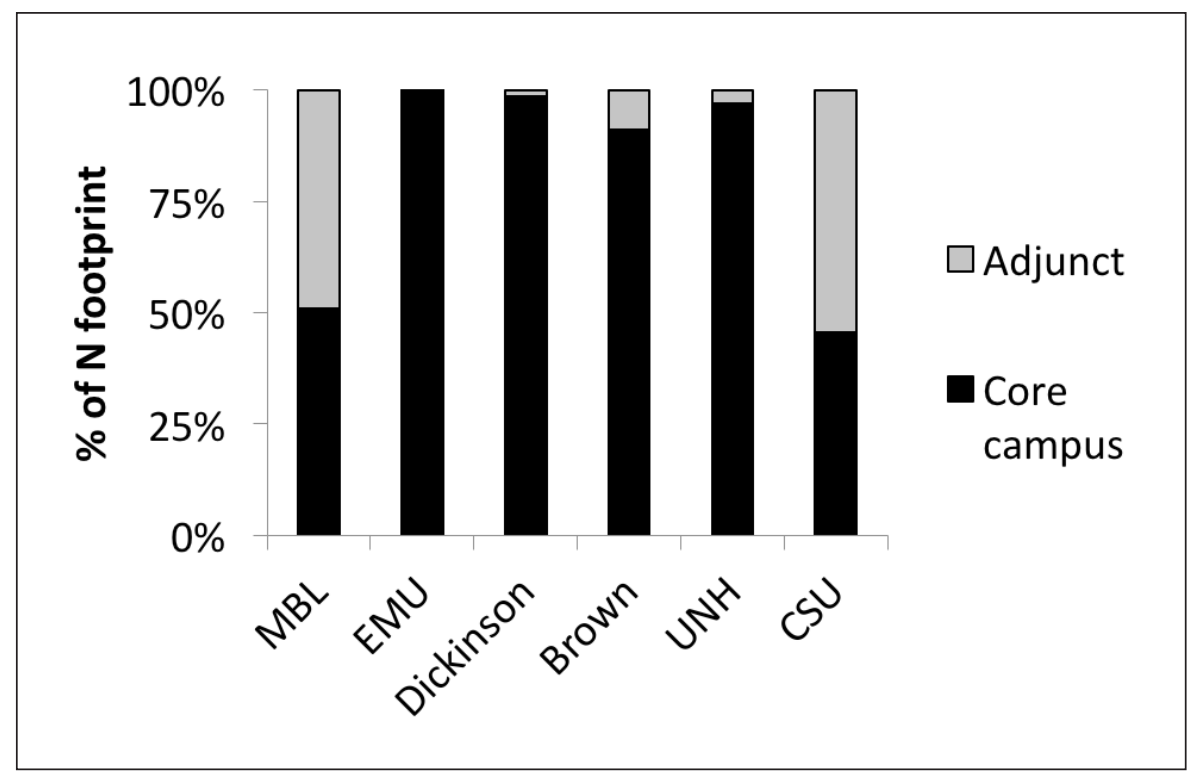

Figure 3. The percent contribution of the core campus footprint and the adjunct footprint for six institutions: the Marine Biological Laboratory (MBL), Eastern Mennonite University (EMU), Dickinson College, Brown University, University of New Hampshire (UNH), and Colorado State University (CSU)

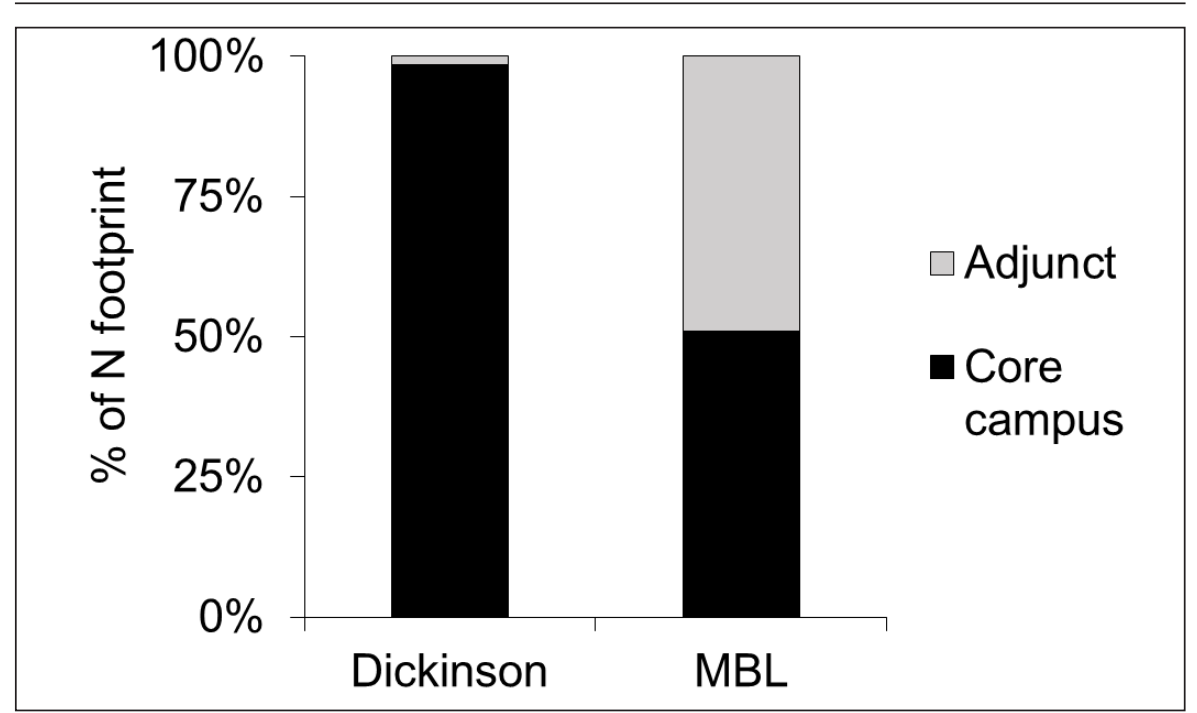

Figure 4. The percent contribution from the core campus footprint and the adjunct footprint: Dickinson College and the Marine Biological Laboratory

though air travel is a small portion of Dickinson's overall footprint, it is a part of their overall educational mission and so to include it is important.

\section{Case Study B: Marine Biological Laboratory and Scientific Research}

The total $\mathrm{N}$ footprint for the Marine Biological Laboratory in 2013 was
7.5 metric tons $\mathrm{N}$. The footprint is based on the campus size, 43,942 gross square meters, which includes cottages that are rented during the summer, but excludes the warehouses and storage facilities in other towns. The cottages were included in the footprint because they are owned by the MBL and are utilized by visiting scientists during the summer season. Also included is a 
dining facility on campus that caters to students, visiting scientists, employees, and visitors. Upstream food production nitrogen losses released 4.0 metric tons $\mathrm{N}$ in 2013, and food consumption released 0.4 metric tons N. Commuting for year-round employees was accounted for, which contributed 0.4 metric tons $\mathrm{N}$ to the overall footprint.

The MBL $\mathrm{N}$ footprint can be divided into core campus activities (food, utilities, transportation) and research-related activities (fertilizer application for scientific experiments, research animals). When accounting only for core activities, the $\mathrm{N}$ footprint was 6.5 metric tons $\mathrm{N}$. However, research activities contribute a significant addition: research animals added 1.0 metric tons $\mathrm{N}$, and a fertilizer application experiment in 2013 added 5.2 metric tons $\mathrm{N}$ (Figure 6). Although the fertilizer experiment lasted for a short period of time, it made up over 40 percent of MBL's $\mathrm{N}$ footprint that year.

The kilograms of nitrogen per person on the MBL campus for food production, food consumption, utilities, and transportation are $3.8,0.32,1.5,0.36$ metric tons $\mathrm{N}$, respectively. However, research increased the use of fertilizer and the number of research animals. Fertilizer increased the nitrogen loss from $0 \mathrm{~kg} \mathrm{~N} /$ person to $4.9 \mathrm{~kg}$ $\mathrm{N} /$ person; research animals increased the nitrogen loss from $0 \mathrm{~kg}$ $\mathrm{N} /$ per person to $0.92 \mathrm{~kg} \mathrm{~N} /$ person. Including research activities is important in accounting for all nitrogen losses associated with an institution's activities.

\section{Other Examples of the Adjunct Footprint}

Two other examples of research activities that can be a large con-

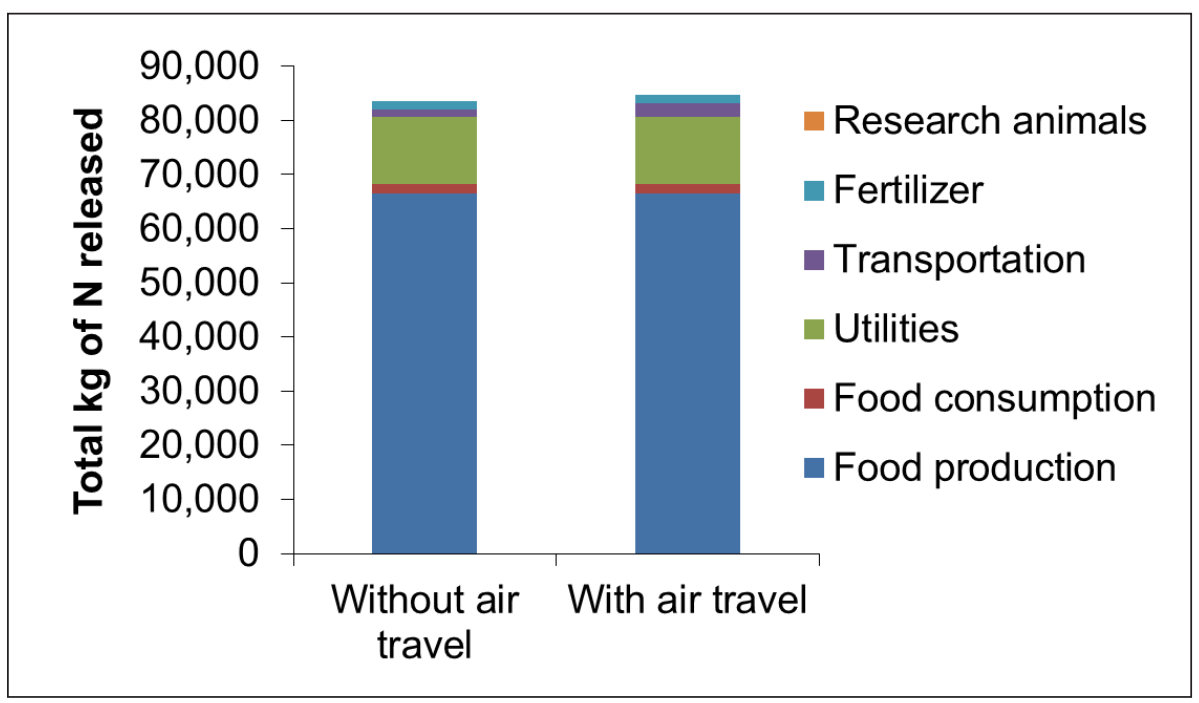

Figure 5. Dickinson College N footprint with and without air travel.

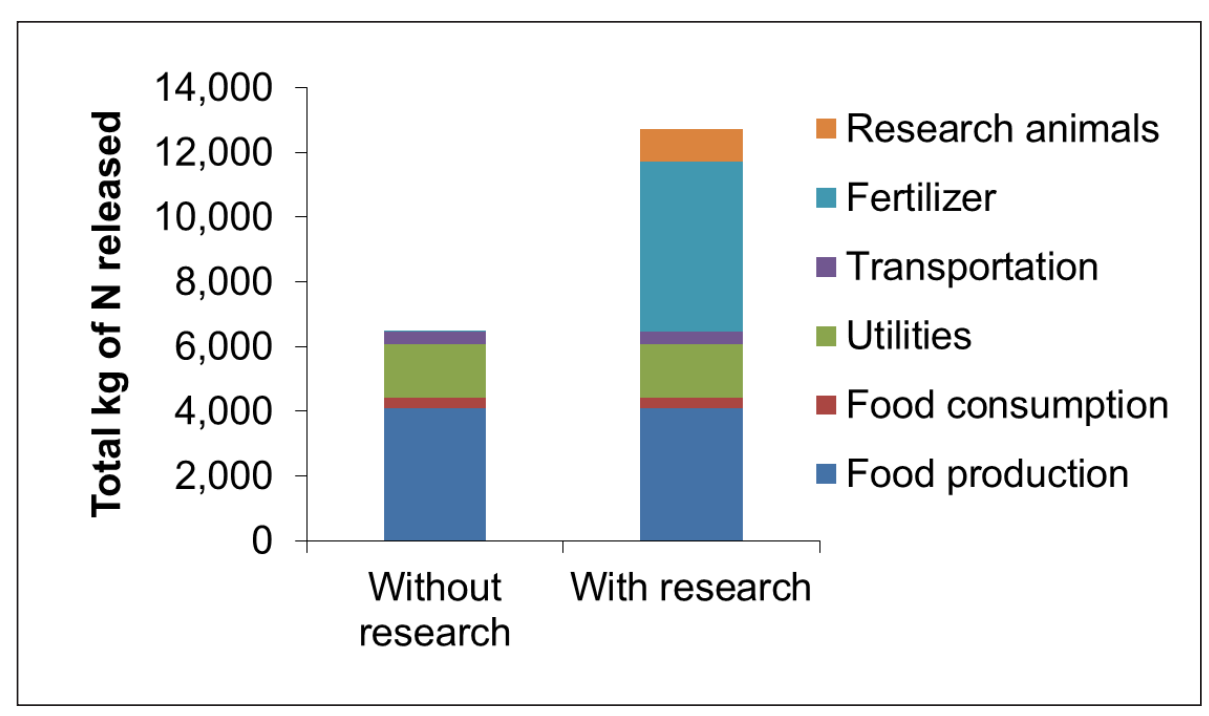

Figure 6. Marine Biological Laboratory N footprint with and without scientific research

tributor to an institution's total $\mathrm{N}$ footprint are agricultural research and a hospital. Colorado State University (CSU) and the University of Virginia (UVA) are institutions that have agricultural research and a hospital, respectively.

CSU has a substantial agricultural research program, which includes multiple agricultural research facilities. Core campus activities make up just 46 percent of CSU's total $\mathrm{N}$ footprint; the remainder (54\%) is a result of agricultural research. ${ }^{16}$ Exclud- ing the agricultural research sector would miss an important sector for nitrogen losses for CSU. However, including agriculture could be misleading when comparing it to other institutions.

UVA has a hospital where its medical students work and it also provides services to the general public. The hospital is included in UVA's carbon footprint. The sectors that could be affected by adding an institution's hospital include food (production and consumption) and 
utilities. Preliminary results indicate that the hospital contributes a large proportion of the UVA $\mathrm{N}$ footprint. UVA includes the hospital in its reduction goals and sustainability efforts; however, because the hospital is not part of UVA's core campus activities, it should not be included in comparisons to other institutions.

\section{Recommendations for Defining System Bounds}

Based on this research, it is recommended that institutions define their core campus footprint system bounds based on their institution's mission. A core campus footprint is a necessary initial start for calculating an institution's $\mathrm{N}$ footprint, but if there is a research component to an institution, then it should be included in the adjunct boundaries for completeness. These decisions should be made for the baseline calculation year of an institution and followed for all additional updates to the $\mathrm{N}$ footprint. If an institution has or intends to calculate its carbon footprint, the same system bounds should be followed for both footprint calculations. The consistency in system bounds for footprints will aid in setting reduction strategies that can reduce both footprints. ${ }^{17}$

A drawback of including research activities is that the total $\mathrm{N}$ footprint will be larger; however, adding research activities results in a more complete picture of an institution's contribution to nitrogen pollution. Another drawback is the difficulty in setting reduction strategies. Potentially, the adjunct footprint could have its own set of reduction strategies, separate from the core campus footprint, which would encourage institutions to think critically about creative ways to reduce their footprints. It is especially important to include a secondary campus when reduction strategies can be applied to the secondary campus. When comparing $\mathrm{N}$ footprints, it is important to compare the core campus footprints so that only comparable activities are included for each institution.

\section{Conclusion}

How institutions define their system boundaries when calculating their $\mathrm{N}$ footprint will impact not only the recorded amount of nitrogen released, but also how the institution will go about setting reduction strategies. Boundaries should represent the institution's mission and focus, and should also follow the same bounds used for a carbon footprint being calculated simultaneously.

\section{Acknowledgments}

The work of the Nitrogen Footprint Tool Network was supported by Cooperative Agreement No. 83563201 awarded by the U.S. Environmental Protection Agency. The data at Dickinson College was collected and analyzed by students in the Center for Sustainability Education and the data from the Marine Biological Laboratory was collected by Sam Kelsey and Kate Morkeski.

\section{Author Disclosure Statement}

No competing financial interests exist.

\section{References}

1. Leach A, Galloway JN, Bleeker A, et al. A nitrogen footprint model to help consumers understand their role in nitrogen losses to the environment. Environ Dev 2012;1:40-66.
2. Galloway, JN, Aber JD, Erisman JW, et al. The nitrogen cascade. Bioscience 2003;53:341-356.

3. Leach A, Majidi AN, Galloway JN, et al. Toward institutional sustainability: A nitrogen footprint model for a university. Sus J Record 2013;6:211-219.

4. Leip A, Weiss F, Lesschen JP, et al. The nitrogen footprint of food products in the European Union. J Agric Sci 2014;152:20-33.

5. Otia A, Malik A, Kanemoto K, et al. Substantial nitrogen pollution embedded in international trade. Nat Geosci 2016;9:111-115.

6. The Climate Registry. General Reporting Protocol for the Voluntary Reporting Program. January 2016. https://www.theclimateregistry.org/ wp-content/uploads/2014/11/General-Reporting-Protocol-Version2.1.pdf. (last accessed 3/21/2017).

7. World Resources Institute and the World Business Council for Sustainable Development. The Greenhouse Gas Protocol: A Corporate Accounting and Reporting Standard, rev. ed. World Resources Institute and the World Business Council for Sustainable Development, Geneva/Washington, DC, 2013. http:// www.ghgprotocol.org/standards/ corporate-standard. (last accessed 3/08/2017).

8. Carbon Management and Analysis Platform (CarbonMAP). http:// campuscarbon.com. (last accessed 10/26/2016).

9. The Clean Air-Cool Planet Campus Carbon Calculator. http:// www.sustainableunh.unh.edu/sites/ sustainableunh.unh.edu/files/images/v6.5_Users_Guide.pdf (last accessed 3/7/2017).

10. Leach A, Majidi A, Galloway JN, et al. How to Calculate Your Institution's Nitrogen Footprint. US Environmental Protection Agency, Washington, DC, 2016. https://cf- 
pub.epa.gov/si/si_public_record_ report.cfm?dirEntryId=315159 (last accessed 3/08/2016).

11. de la Reguera E. The nitrogen footprint of Dickinson College. Senior thesis, Dickinson College, 2014. 12. Leary N, de la Reguera E, and Fitzpatrick $S$. Reducing the nitrogen footprint of a small residential college. Sus J Record 2017;10:96-104. 13. Notopolos M. Toward Institutional Sustainability-A Nitrogen Footprint for the Marine Biological Laboratory. 2012. http://www. mbl.edu/ses/files/2014/09/Noto-
poulos-Maggie.pdf (last accessed 3/08/2017).

14. Castner EA, AM Leach AM, N Leary N, et al. The Nitrogen Footprint Tool Network: A multi-institution program to reduce nitrogen pollution. Sus J Record 2017;10:79-88. 15. Dickinson College Global Philosophy. http://www.dickinson.edu/ homepage/576/about. (last accessed 3/08/2017).

16. Kimiecik J, Baron J, Taylor E, et al. Adding a nitrogen footprint to Colorado State University's sustainability plan. Sus J Record 2017;10:88-95.
17. Leach AM, Galloway JN, Castner EA, et al. An integrated tool for calculating and reducing institution carbon and nitrogen footprints. Sus J Record 2017;10:140-148.

Address correspondence to: Elizabeth de la Reguera Marine Biological Laboratory

The Ecosystems Center

$7 \mathrm{MBL}$ Street

Woods Hole, MA 02543

E-mail: elreguera@gmail.com 